\title{
Protection of FK506 against neuronal apoptosis and axonal injury following experimental diffuse axonal injury
}

\author{
TING-QIN HUANG ${ }^{1}$, JIN-NING SONG $^{1}$, FENG-WEI ZHENG ${ }^{2}$, \\ HONG-GANG PANG ${ }^{1}$, YONG-LIN ZHAO ${ }^{1}$, HUA GU ${ }^{1}$ and JUN-JIE ZHAO ${ }^{1}$ \\ ${ }^{1}$ Department of Neurosurgery, The First Affiliated Hospital of Xi'an Jiaotong University, Xi'an, Shaanxi 710061; \\ ${ }^{2}$ Department of Neurosurgery, The Second Hospital of Lanzhou University, Lanzhou, Gansu 730030, P.R. China
}

Received January 18, 2016; Accepted February 7, 2017

DOI: $10.3892 / \mathrm{mmr} .2017 .6350$

\begin{abstract}
Diffuse axonal injury (DAI) is the most common and significant pathological features of traumatic brain injury (TBI). However, there are still no effective drugs to combat the formation and progression of DAI in affected individuals. FK506, also known as tacrolimus, is an immunosuppressive drug, which is widely used in transplantation medicine for the reduction of allograft rejection. Previous studies have identified that FK506 may play an important role in the nerve protective effect of the central nervous system. In the present study, apoptosis of neuronal cells was observed following the induction of experimental DAI. The results demonstrated that it was closely related with the upregulation of death-associated protein kinase 1 (DAPK1). It was hypothesized that FK506 may inhibit the activity of DAPK1 by inhibiting calcineurin activity, which may be primarily involved in anti-apoptosis following DAI induction. Through researching the expression of nerve regeneration associated proteins (NF-H and GAP-43) following DAI, the present study provides novel data to suggest that FK506 promotes axon formation and nerve regeneration following experimental DAI. Therefore, FK506 may be a potent therapeutic for inhibiting nerve injury, as well as promoting the nerve regeneration following DAI.
\end{abstract}

\section{Introduction}

Diffuse axonal injury (DAI) is the most common pathological feature of traumatic brain injury (TBI) due to high mortality and morbidity rates following injury (1). DAI typically results from head rotational acceleration/deceleration following impact, and is characterized by swellings or varicosities of

Correspondence to: Professor Jin-Ning Song, Department of Neurosurgery, The First Affiliated Hospital of Xi'an Jiaotong University, 277 Yanta West Road, Xi'an, Shaanxi 710061, P.R. China E-mail: jinningsong@126.com

Key words: FK506, death-associated protein kinase 1, diffuse axonal injury, growth-associated protein- 43 , neurofilament-H axons and large terminal bulbs $(2,3)$. The clinical manifestation of DAI in the long term includes: Disorders of consciousness, difficult clinical diagnosis and bad prognosis, often resulting for vehicle collisions, blast exposure and falls $(4,5)$. Although diffusion tensor imaging (DTI) has long been regarded as a valuable technique for evaluating the white matter injury following DAI in clinic (6-8), there remain no suitably effective drugs to attenuate the formation and progression of DAI in patients.

Neuronal apoptosis is a major contributor to the secondary neuronal injury following TBI through multiple cellular mechanisms, including $\mathrm{Ca}^{2+}$ overload, production of reactive oxygen/nitrogen species (9-12) and glutamate-mediated excitotoxicity $(13,14)$. Axonal injury is a common consequence of TBI (15). The characteristic axonal pathological changes include axonal swelling and distortion, the formation of axonal bulbs, and axotomy $(16,17)$. Therefore, treatment with an agent that has the ability to inhibit the resulting neuronal apoptosis and axonal injury may potentially improve multiple aspects of the response of the brain to trauma.

FK506 (also known as tacrolimus) is an immunosuppressive drug, frequently used in the field of transplantation medicine in order to reduce allograft rejection. Using a middle cerebral artery occlusion model, the neuroprotection of FK506 was demonstrated to act via inhibition of apoptotic and necrotic cell death, suppression of microglia activation and alterations in cytokine expression, including interleukin (IL)-1 $\beta$, IL-6 and tumor necrosis factor (TNF)- $\alpha$ (18). A previous study identified that FK506 may be able to inhibit neuronal apoptosis by maintaining $\mathrm{Bcl}-2$-associated agonist of cell death protein turnover, inhibiting cytochrome $\mathrm{C}$ release from mitochondria (19), and inhibiting the release of arachidonic acid (20). In addition, studies have demonstrated that FK506 may attenuate the axonal injury and improve axonal survival or regeneration by inhibiting the calcineurin (CaN) activity or the post-traumatic compound action potential after TBI (21-23).

Death-associated protein kinase 1 (DAPK1) is a novel and specific cell death signaling molecule, which is directly linked to glutamate receptor channels (24). It is a newly-identified $\mathrm{Ca}^{2+} /$ calmodulin-dependent serine/threonine protein kinase (25) that serves a role in several modes of cell death, including apoptosis and autophagy (26). 
Growth-associated protein-43 (GAP-43) is a specific phosphatase protein in the vertebrate nerve cell membrane, which is closely related to neural development, axonal plasticity and synaptic remodeling (27). Several studies have demonstrated that GAP-43 is upregulated in nerve regeneration (28) and plasticity following TBI in rat models (29). Neurofilament-H (NF-H) is the most abundant protein component of neurons and is released in large amounts from damaged or dying neurons (30). NF-H is relatively simple to detect as a predictive biomarker of the outcome following TBI (31). Furthermore, $\mathrm{NF}-\mathrm{H}$ only exists in the axons, not in the neuronal cell bodies in physiological condition. Following TBI, the neuronal cells synthesize a large amount of NF-H, to adapt to the need of nerve regeneration. Therefore, it could not only reflect the neuronal function, but also reflect the situation of neurite regeneration $(30,31)$.

As a result of previous studies, the present study hypothesized that FK506 may be therapeutically efficacious in neuronal apoptosis and axonal injury following ischemia or nerve trauma. To the best of our knowledge, the present study is the first to have examined the possible functional role of FK506 in neuronal apoptosis or regeneration following experimental DAI. Neuronal apoptosis and axonal pathological alterations were examined in a rat model, following induction of DAI via lateral head rotation trauma. Furthermore, the protective effects of FK506 on brain injury following DAI were investigated.

\section{Materials and methods}

Model of DAI in vivo and treatments. A total of 90 healthy, adult male Sprague-Dawley rats (weight, 250-300 g, 8-10 weeks of age), with the same genetic background, were obtained from the Animal Experimental Center of Medical College of Xi'an Jiaotong University (License number SCXK (Shaanxi) 2007-001). All animals received humane care in compliance with the Guide for the Care and Use of Laboratory Animals from the National Institutes of Health (publication no. 80-23). Experimental and surgical procedures, as well as post-operative care were each approved by the Biomedical Ethics Committee of Medical College of Xi'an Jiaotong University (Xi'an, China). Animals were housed and fed in a temperature- and humidity-controlled environment with a standardized light-dark cycle (12 h/12 h). Food and water were available ad libitum.

Rats were randomly divided into three groups: A control group (Sham group, $n=30$ ), a DAI/vehicle-treated group (DAI+Vehicle group, $n=30$ ) and a DAI/FK506-treated group (DAI+FK506 group, n=30). Each of the groups was randomly divided into three subgroups that represented 1, 3 and 7 days post-injury (PI). The present study involved the establishment of a DAI model in rats by lateral head rotation device (32), which was modified from the Xiaosheng et al $(33,34)$. All rats in DAI+Vehicle and DAI+FK506 groups after weighting were anesthetized by intraperitoneal injection of chloral hydrate (30 $\mathrm{mg} / \mathrm{kg}$ ) and placed in the prone position. Following anesthesia, the head of the rats were fixed in the rat instant head rotating injury device, the rat head was horizontally secured to the lateral head rotation device by two lateral ear bars, a head clip and an anterior teeth hole, with its body $30^{\circ}$ oblique to the top of the laboratory table. For the injury group, following pushing the trigger, the device rapidly rotated the rat head through a $90^{\circ}$ angle laterally (i.e., in the coronal plane). The rats were placed in separated cages, maintaining the room temperature between 18 and $26^{\circ} \mathrm{C}$ and the indoor relative humidity at 40-70\%. Primary coma was observed in all injured rats. Rats that succumbed to their injuries were excluded and later replaced by new rats. Control rats (Sham group) only underwent anesthesia and were fixed to the device, but were not subjected to injury.

Animals also received either FK506 or a vehicle $(0.9 \%$ sterile saline) delivered intravenously $30 \mathrm{~min}$ pre-DAI. A single $3 \mathrm{mg} / \mathrm{kg}$ of FK506 in $0.9 \%$ sterile saline to a total volume of $1.0 \mathrm{ml}$ was infused over a $10 \mathrm{~min}$ period to ensure that the rate of injection did not significantly elevate $\operatorname{MABP}(21,22)$. The vehicle was administrated using the same protocol. FK506 (Tacrolimus) was purchased from Abcam (Cambridge, UK; cat. no. ab120223).

Embedding and sectioning. Euthanasia was conducted at 1, 3 and 7 days post-injury following being freed from the injury device. Rats in the Sham-operated group were euthanized at the same times. Half of the rats $(n=45)$ were sacrificed and perfused with $250 \mathrm{ml}$ of normal saline only. The brain stem and the hippocampus were collected for western blotting. The remaining rats $(n=45)$ were sacrificed and perfused with $250 \mathrm{ml}$ of normal saline followed by $400 \mathrm{ml}$ of $4 \%$ paraformaldehyde in 0.01 M PBS. The whole brain removed and post-fixed in $4 \%$ paraformaldehyde solution, dehydrated via a graded ethanol series, vitrified with dimethyl benzene, embedded with paraffin and sectioned into $10 \mu \mathrm{m}$ thick sections using a microtome. A total of five sections, including the hippocampus tissue and brain stem tissue from each animal, were randomly chosen and mounted on poly-L-lysine coated slides (cat. no. P4981; Thermo Fisher Scientific, Inc., Waltham, MA, USA) for Glees-Marsland Silver staining, terminal deoxynucleotidyl transferase dUTP nick end labeling (TUNEL) staining, immunofluorescence and immunohistochemical staining.

Immuohistochemistry. The brain sections were deparaffinized in xylene and hydrated in a decreasing gradient of alcohol to distilled water. Endogenous peroxidase activity was blocked with $3 \% \mathrm{H}_{2} \mathrm{O}_{2}$ for $5 \mathrm{~min}$, followed by a brief rinse in distilled water and a 15 min wash in PBS. Sections were placed in $0.01 \mathrm{~mol} / 1$ citrate buffer $(\mathrm{pH} 7.2)$ and heated in a microwave oven at $95^{\circ} \mathrm{C}$ for $30 \mathrm{~min}$. Sections were cooled at room temperature for $20 \mathrm{~min}$ and rinsed again in PBS. Non-specific protein binding was blocked by $30 \mathrm{~min}$ of incubation in normal goat serum (cat. no. 16210064; Gibco; Thermo Fisher Scientific, Inc.) at room temperature, followed by incubation with primary antibodies: Rabbit anti-DAPK1 monoclonal antibody (dilution, 1:500; cat. no. 3798-1; Epitomics, Burlingame, CA, USA), mouse anti-NF-H monoclonal antibody (dilution, 1:200; cat. no. 2836; Cell Signaling Technology, Inc., Danvers, MA, USA) and mouse anti-GAP-43 monoclonal antibody (dilution, 1:500; cat. no. sc-33705; Santa Cruz Biotechnology, Inc., Dallas, TX, USA) for $24 \mathrm{~h}$ at $4^{\circ} \mathrm{C}$, followed by a 15 min wash in PBS. Sections were then incubated with goat anti-rabbit (dilution, 1:200; cat. no. 31460; Thermo Fisher Scientific, Inc.) or 
goat anti-mouse IgG-biotin (dilution, 1:200; cat. no. 31431; Thermo Fisher Scientific, Inc.) for $30 \mathrm{~min}$ at $37^{\circ} \mathrm{C}$, and sections were washed with PBS for $15 \mathrm{~min}$ following each step. Diaminobenzidine was used as the chromogen, and hematoxylin was used as the counterstain. Sections incubated with PBS in the absence of primary antibodies were used as negative controls. Microscopic observation of the stained sections was performed by an experienced pathologist blind to the experimental conditions. The immunoreactivity of all of the molecular markers was analyzed using Image-Pro Plus 6.0 software (Media Cybernetics, Inc., Rockville, MD, USA) in five microscopic fields (magnification, x200).

Glees-Marsland Silver staining. The brain paraffin-embedded sections at 1 day post-injury were examined by Glees-Marsland Silver staining methods as previously described $(35,36)$. Nerve fibers were stained black. In each slice, five microscopic fields (magnification, x400) were randomly selected and their images captured.

TUNEL staining. The brain paraffin-embedded sections were also examined by TUNEL assay using the DeadEnd colorimetric TUNEL system detection kit (cat. no. G7130; Promega Corporation, Madison, WI, USA) as described previously (37). Brain tissue sections were initially deparaffinized with xylene, rehydrated through descending concentrations of ethanol and rinsed for $15 \mathrm{~min}$ in $0.1 \mathrm{M} \mathrm{PBS}$ and then treated with $20 \mu \mathrm{g} / \mathrm{ml}$ of Proteinase $\mathrm{K}$ for $20 \mathrm{~min}$ at room temperature. Samples were treated with $3 \% \mathrm{H}_{2} \mathrm{O}_{2}$ in methanol for $20 \mathrm{~min}$ to inactivate endogenous peroxidase. Following washing with PBS, specimens were incubated in the rTdT reaction mixture $(100 \mu \mathrm{l}$; combining $98 \mu \mathrm{l}$ of Equilibration Buffer, $1 \mu \mathrm{l}$ of Biotinylated Nucleotide Mix and $1 \mu \mathrm{l}$ of rTdT Enzyme) at $4^{\circ} \mathrm{C}$ overnight. Following incubation, all the sections were rinsed in PBS and incubated with horseradish peroxidase (dilution, 1:500) for $30 \mathrm{~min}$ at room temperature. Then, the sections were washed extensively with PBS for $5 \mathrm{~min}$ and treated with DAB solution (30 mg DAB and $200 \mu 1 \mathrm{H}_{2} \mathrm{O}_{2} / 100 \mathrm{ml} \mathrm{PBS}$ ) for $10 \mathrm{~min}$ at room temperature in the dark. Following washing under running water, all the sections were counterstained with hematoxylin for $30 \mathrm{sec}$. Finally, the sections were dehydrated in increasing graded ethanol, cleared in xylene and mounted with a cover slip. With this method, apoptotic nuclei were identified by the presence dark brown staining.

Immunofluorescence staining. The brain tissue sections were de-paraffinized in xylene and hydrated in a decreasing gradient of alcohol to distilled water as above. Then sections were blocked with $10 \%$ normal serum blocking solution species the same as the secondary antibody, containing $3 \%$ (w/v) BSA, $0.1 \%$ Triton X-100, and 0.05\% Tween-20 $2 \mathrm{~h}$ at room temperature in order to avoid unspecific staining. The brain sections were incubated with primary antibodies for anti-NF-H mouse monoclonal antibody (dilution, 1:400; cat. no. 2836; Cell Signaling Technology, Inc.) overnight at $4^{\circ} \mathrm{C}$. Then sections were incubated with fluorescein isothiocyanate conjugated (FITC)-secondary antibody (goat anti-mouse; 1:500, cat. no. A24513; Invitrogen; Thermo Fisher Scientific, Inc.) for $2 \mathrm{~h}$ at room temperature. Sections were covered with DAPI (0.1 mg/ml in PBS; cat. no. ab104139; Abcam, Cambridge, MA, USA) for $40 \mathrm{~min}$ at room temperature and subsequently examined with a Leica fluorescence microscope (DM5000B; Leica Microsystems GmbH, Wetzlar, Germany).

Western blot analysis. Total protein was isolated from rat brain stem and hippocampus tissues using ice-cold radioimmunoprecipitation assay buffer (cat. no. 89900; Thermo Fisher Scientific, Inc). Total protein concentrations were measured using the BCA Protein Assay kit (cat. no. 23229; Thermo Fisher Scientific, Inc.). Samples (60 $\mu \mathrm{g} / \mathrm{lane})$ were separated on an SDS gel (8\% for DAPK1 or $10 \%$ for GAP43 and $\beta$-actin) and electrotransferred onto polyvinylidene difluoride membranes. Following incubation for $2 \mathrm{~h}$ in a blocking solution [5\% non-fat milk in $20 \mathrm{mM}$ Tris- $\mathrm{HCl}$, $150 \mathrm{mM} \mathrm{NaCl}, 0.1 \%$ Tween-20 (TBST)], membranes were blotted with primary antibodies against DAPK1 (dilution, 1:1,000; cat. no. 3798-1; Epitomics), GAP-43 (dilution, 1:1,000; cat. no. sc-33705; Santa Cruz Biotechnology, Inc.) or $\beta$-actin (dilution, 1:1,000; cat. no. ab8226; Abcam) overnight at $4^{\circ} \mathrm{C}$. Following extensive washing with TBST buffer, membranes were incubated with HRP-conjugated anti-rabbit/mouse secondary antibodies (1:1,000; cat. no. 31460/31430; Thermo Fisher Scientific, Inc.) for $1 \mathrm{~h}$ at room temperature. Specific bands were detected using a ECL Western Blotting Detection System (cat. no. WBKLS0100; EMD Millipore, Billerica, MA, USA). Western blotting was performed in at least six independent experiments. Membranes were scanned and the density of the bands analyzed with Quantity one software (version 4.62; Bio-Rad Laboratories, Inc., Hercules, CA, USA).

Statistical analysis. SPSS software (version, 18.0; SPSS Inc., Chicago, IL, USA) was used for statistical analyses. All data were presented as mean \pm standard error of the mean $(n=5)$. Comparisons among multiple groups were performed using one-way analysis of variance. Comparisons between the two groups were performed using Fisher's Least Significant Difference test. $\mathrm{P}<0.05$ was considered to indicate a statistically significant difference.

\section{Results}

General observation. No animals succumbed to the treatments in the Sham groups, and no obvious neurological deficits were observed. However, a series of neurological symptoms had been discovered in the rats following DAI, including the disturbance of consciousness, reduction of physical activity, a weakened response to stimulation, drowsiness, instability in walking, weakened balance ability, loss of weight and even paralysis of limbs. Both Glees-Marsland silver staining and immunocytochemical experiments for NF-H demonstrated well changes of the axonal pathology. The rat brain tissue of the normal control group under the light microscope demonstrated that the nerve axons were smooth, with uniform thickness and arranged closely in the cerebral white matter, however the DAI model group in cerebral white matter present visible tortuous and swollen axons, even the axonal fracture and axonal ball formation (Fig. 1A and B). These results suggested that the DAI model was successfully established, 
A

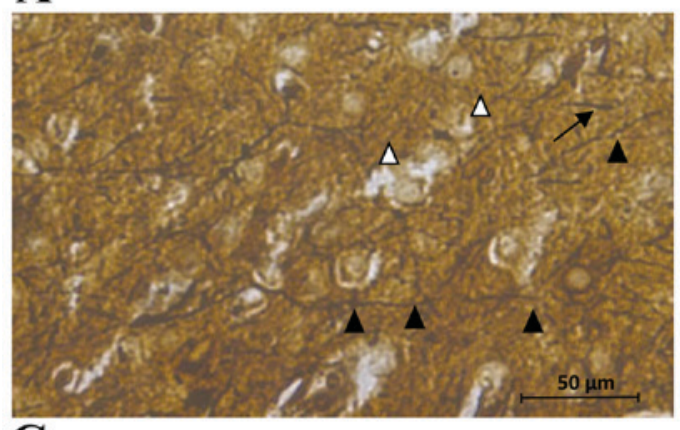

C

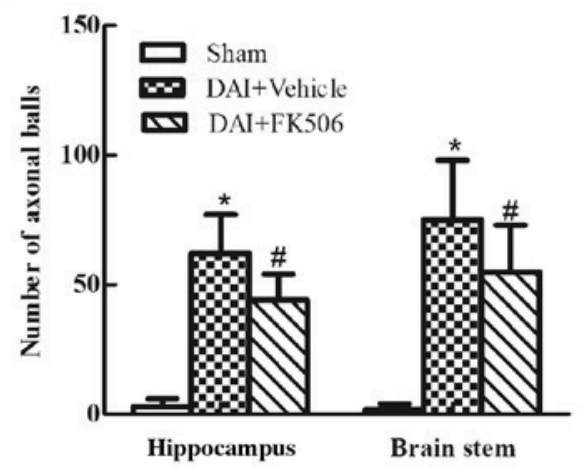

B
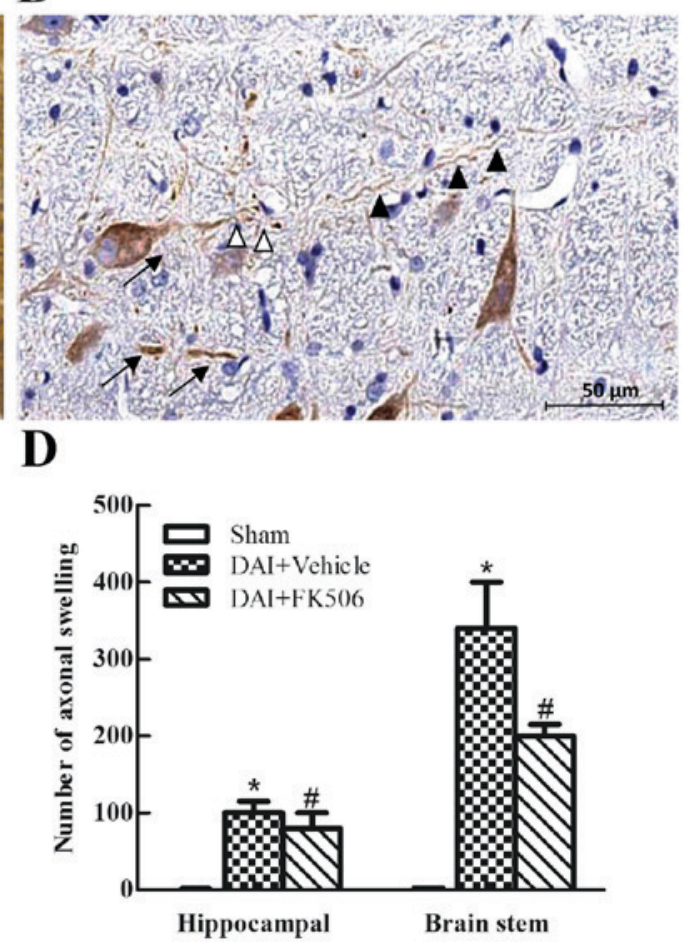

Figure 1. Immunohistochemical and Glees-Marsland staining of the hippocampal CA1 region and brainstem. The axons became varicose, swollen and presented the formation of axonal retraction balls in the injured group (magnification, x400). (A) The axonal pathological alterations following DAI, as conducted by Glees-Marsland silver staining (white arrowheads, axonal retraction balls; black arrowheads, axonal varicose; black triangles, axonal swelling). (B) The axonal pathological alterations following DAI as conducted by immunohistochemical staining on NF-H (white arrowheads, axonal retraction balls; black arrowheads, axonal varicose; black triangles, axonal swelling). (C) Bar chart presenting the number of axonal retractions balls in the field of view of the hippocampal CA1 region and brainstem in Sham, DAI+Vehicle and DAI+FK506 groups. (D) Bar chart presenting the amount of axonal swelling in the field of view of the hippocampal CA1 region and brainstem in Sham, DAI+Vehicle and DAI+FK506 groups. "P<0.05 vs. Sham group; ${ }^{*} \mathrm{P}<0.05$ vs. DAI+Vehicle group. DAI, diffuse axonal injury; NF-H, neurofilament-H.

allowing its use in the subsequent experiment. In addition, the pathological changes in axons were attenuated significantly in the FK506 group when compared with the DAI+Vehicle group (both $\mathrm{P}<0.05$; Fig. $1 \mathrm{C}$ and D).

Immunocytochemical analysis of DAPK1. The expression of DAPK1 through immunohistochemical analysis was examined (Fig. 2). The results demonstrated that DAPK1 was primarily localized to the cytoplasm of the hippocampal CA1 and brain stem regions. DAPK1 exhibited low expression in the Sham-treated group (Fig. 2A and D). In the DAI+Vehicle group, the positive cell number of DAPK1 was significantly increased at $24 \mathrm{~h}$ post-injury when compared with the Sham group, reaching a peak at day 3 , before decreasing at day 7 (all $\mathrm{P}<0.05$; Fig. 2B, E, G and H). The intervention by FK506 significantly decreased the positive cell number of DAPK1 at each time point, when compared with the DAI+Vehicle group (all $\mathrm{P}<0.05$; Fig. 2C, F, G and H).

TUNEL Staining. TUNEL staining labels apoptotic cells directly, with normal cells stained in blue, and apoptotic cells stained in brown (Fig. 3). The results indicated that there were $\sim 0$ positive neurons in the hippocampus and brain stem of the Sham group (Fig. 3A and D). The number of TUNEL-positive cells in the DAI+Vehicle group was increased compared with the Sham groups (Fig. 3B, E and G), but decreased in the FK506 group (Fig. 3C, F and G).
Immunocytochemical analysis for $N F-H$. The expression of NF-H was evaluated through immunohistochemical and immunofluorescence staining (Fig. 4). The results indicated that NF-H was mainly localized in the axons in the hippocampal CA1 region, as well as the brain stem region. NF-H was lowly expressed in the Sham-treated group (Fig. 4A, D, G, J and $\mathrm{M}$ and $\mathrm{N}$ ). Following DAI, the expression of positive NF-H was increased and to peak at 1 day post-injury, then gradually reduced at days 3 and 7 (Fig. 4B, E, H, K, M and N). However, following the intervention of FK506, this may increase the expression of positive NF-H at each time point, and could even be able to maintain a high-level expression of NF-H at day 3 post-injury (Fig. 4C, F, I, L, M and N).

Expression of GAP-43 after DAI. Western blot analysis revealed a significant upregulation of GAP-43 in the DAI group when compared with the control and Sham groups (Fig. 5). In addition, a gradual increase of GAP-43 expression was observed until it reached a peak 7 days post-injury in the hippocampus and following DAI. When FK506 was administered, western blot analysis revealed that FK506 induced a upregulation of GAP-43 expression when compared with the levels detected in the DAI and vehicle group (Fig. 5A, B, I and J). The expression of GAP-43 in the normal rat brain was low, as demonstrated by immunohistochemistry staining. Following DAI, there was observation of GAP-43 protein assembled in the membrane of neuronal cells and neuropil, and the expression of GAP-43 

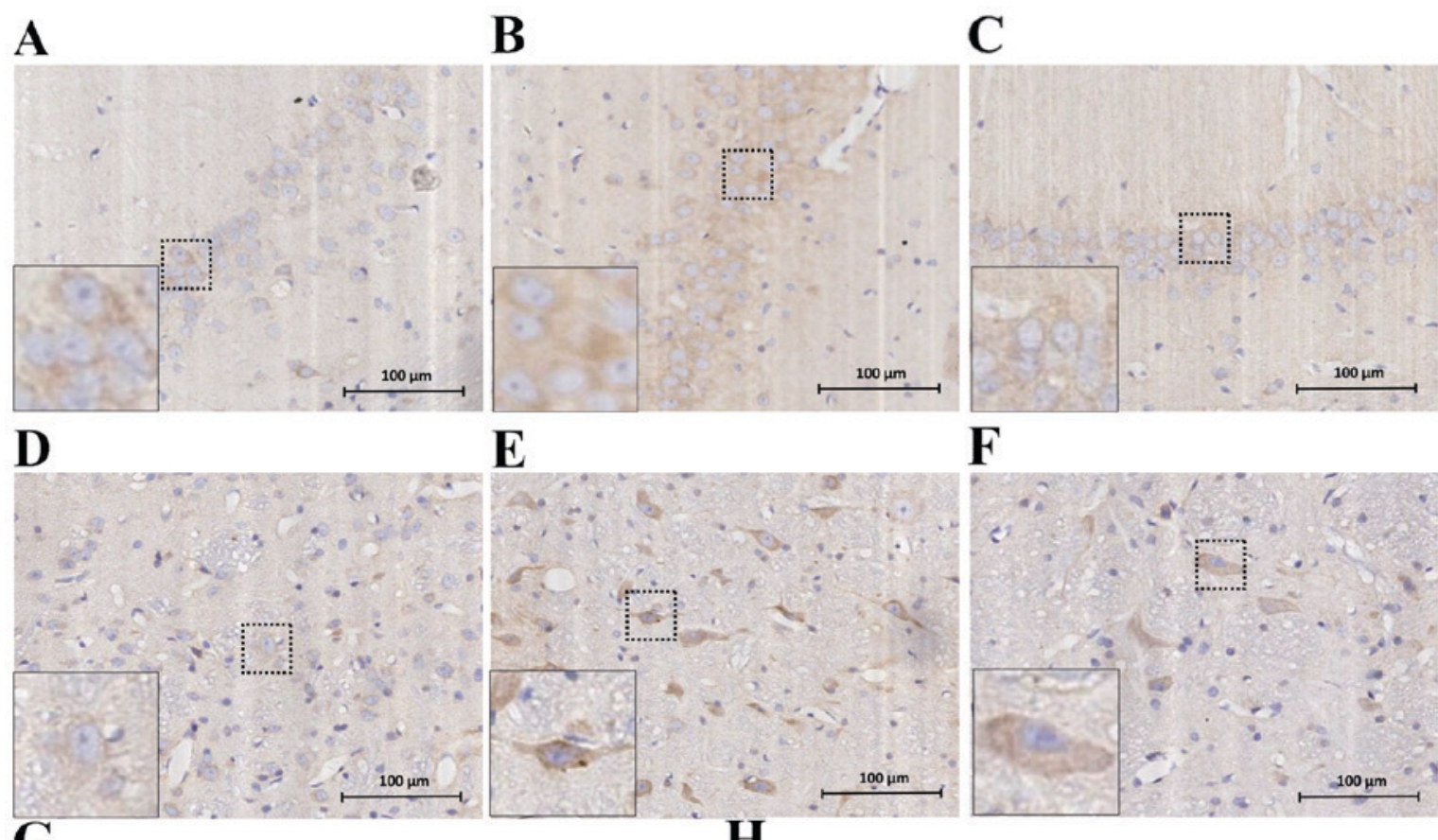

E
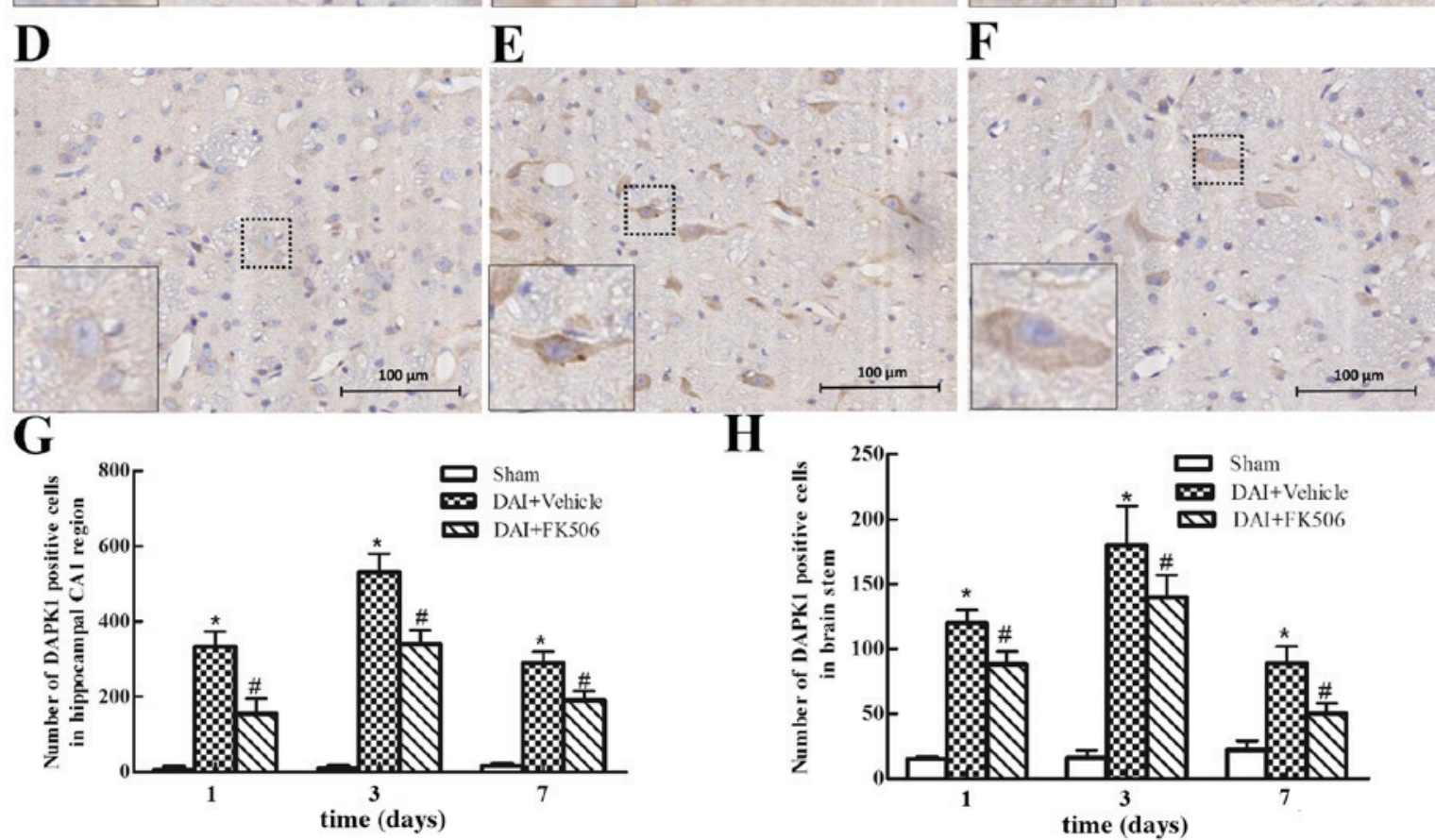

H

$100 \mu \mathrm{m}$

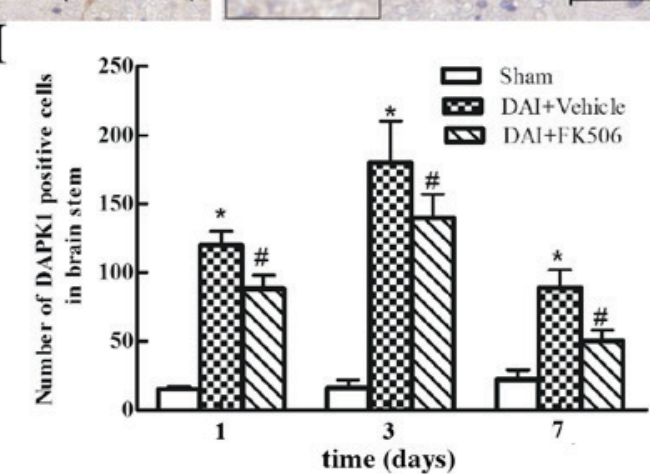

Figure 2. Immunohistochemistry of DAPK1 in the hippocampal CA1 region and brainstem (magnification, x200). Photographs of DAPK1 immunohistochemistry staining in the hippocampal CA1 region in (A) Sham, (B) DAI+Vehicle and (C) DAI+FK506 groups. Photographs of DAPK1 immunohistochemistry staining in the brainstem in (D) Sham, (E) DAI+Vehicle and (F) DAI+FK506 groups. (G) Quantitative analysis of the number of DAPK1-positive cells/mm² in the hippocampal CA1 region in Sham, DAI+Vehicle and DAI+FK506 groups at each time point. (H) Quantitative analysis of the number of DAPK1-positive cells $/ \mathrm{mm}^{2}$ in the brainstem in Sham, DAI+Vehicle and DAI+FK506 groups at each time point. ${ }^{*} \mathrm{P}<0.05 \mathrm{vs}$. Sham group; ${ }^{\text {P }}<0.05$ vs. DAI+Vehicle group. DAPK1, death-associated protein kinase 1; DAI, diffuse axonal injury.

was highest at day 7 in the hippocampus and at day 3 in the brainstem. In addition, administration of the FK506 caused the peak of the expression to advance to day 1 post-injury and maintained a high-level of expression at day 3 (Fig. 5C-H).

\section{Discussion}

In DAI, the axonal degeneration resulted from the stretching and shearing force, caused by the rapid and sudden acceleration or deceleration of the brain tissue during a traumatic event. The secondary injury manifests as a series of dynamic metabolic imbalances, including loss of ionic homeostasis, injury of reactive oxygen, production of excitotoxicity and overload of $\mathrm{Ca}^{2+}$, which ultimately resulted in neuronal cell death and axonal injury $(10,32,38,39)$. DAI in the brain may cause neurologic and cognitive impairments, reflected in consciousness and personality alteration. There have been few treatments that have been proven to be effective for DAI patients (38). In the present study, the molecular mechanism underlying the neuroprotective actions of FK506 was investigated, examining the neuronal cell death and axonal injury in the affected rat brain. The DAI model for the current study was based on the DAI modeling device developed by Xiaosheng et al $(33,34)$, which was established by instant rotational acceleration (32). In addition, axonal pathological changes in the hippocampus and brainstem were observed using Glees-Marsland silver staining and immunohistochemistry staining of NF-H. Specific pathological alterations were observed in axons associated with DAI, including nerve axonal varicose, swelling, fracture and retraction. The reticular formation of brainstem has the function of maintaining the biological consciousness, so the degree of axonal injury in the DAI-affected brain may seriously affect the degree of consciousness, which is consistent with the severity of the disorder in the clinic. However, in the present study, FK506 was identified to significantly reduce the axonal pathological changes following DAI, which may be associated with the activity of CaN and MPT (21).

It has been recognized that apoptotic cell death serves a key role in neuronal cell death in TBI $(40,41)$, and that limiting the apoptotic cascades following DAI may result 

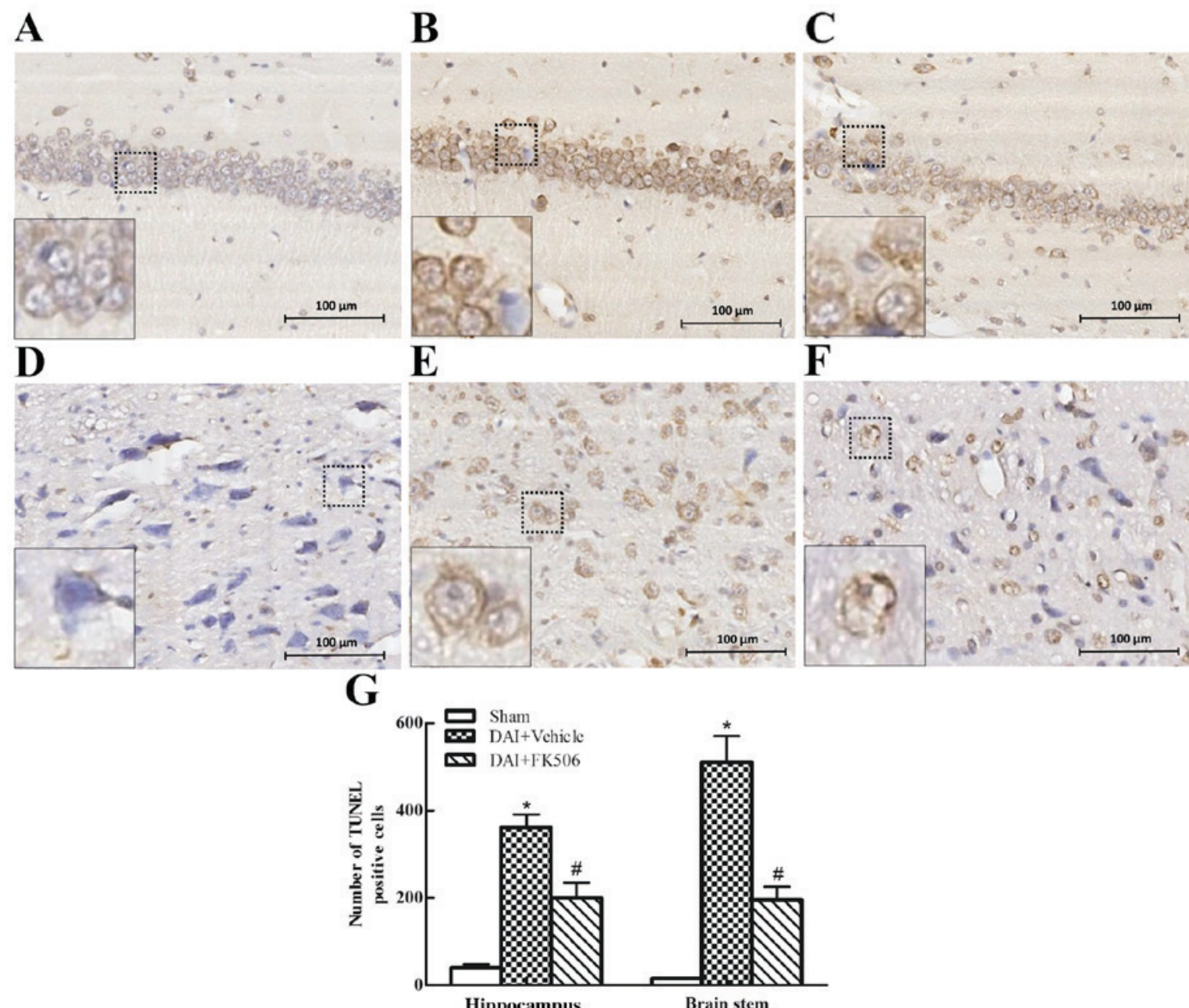

Figure 3. TUNEL staining in the hippocampal CA1 region and brainstem (magnification, x200). Photographs of TUNEL staining in the hippocampal CA1 region in the (A) Sham, (B) DAI+Vehicle and (C) DAI+FK506 groups. Photographs of DAPK1 immunohistochemistry staining in the brainstem in (D) Sham, (E) DAI+Vehicle and (F) DAI+FK506 groups. (G) Quantitative analysis of the number of TUNEL-positive cells/mm² in the hippocampal CA1 region and brainstem in Sham, DAI+Vehicle and DAI+FK506 groups at 3 days post-injury. ${ }^{*} \mathrm{P}<0.05$ vs. Sham group; ${ }^{\#} \mathrm{P}<0.05$ vs. DAI+Vehicle group. TUNEL; terminal deoxynucleotidyl transferase dUTP nick end labeling; DAI, diffuse axonal injury.

in decreased mortality rates, improve functional outcome scores, shorter hospital stays and decrease the charges for healthcare $(10,38,39)$. In the present study, the aim was to evaluate the effect of FK506 on the apoptotic cell death pathway following DAI, the expression of DAPK1 was analyzed, and measured the number of TUNEL-positive cells in the hippocampal CA1 region and brainstem. The authors identified that the expression of DAPK1 was significantly increased following DAI, and the apoptosis of neurons in hippocampus and brainstem was significantly increased at the same time. Previous studies indicated that DAPK1 serves a significant role in several modes of cell death, including apoptosis and autophagy (26). The apoptosis induced by DAPK1 is involved in Fas- (42), interferon- $\gamma$-, TNF- (43), ceramide- $(26)$, and p53- $(44,45)$ mediated apoptosis, as well as in the disruption of matrix survival signals and suppression of integrin-mediated cell adhesion. DAPK1 appeared to function early in the apoptotic pathway prior to the commitment of the cells to apoptosis. In the developing and adult central nervous system, DAPK1 mRNA is widely expressed in proliferative regions within the cerebral cortex and hippocampus (43). In addition, inhibition of DAPK1 with a selective inhibitor attenuates hypoxia-ischemia-induced acute brain injury (45). DAPK1 controls a range of key signaling and cell death pathways as a molecular switch, and also has been suggested to have an important role in excitotoxicity (45-47). Emerging evidence suggests that inhibition of DAPK1, which prevents excessive NMDA receptor (R) activation without interfering with physiological functions, provides neuroprotection in animal models of adult stroke $(24,48)$. NMDAR activation influences neuronal proliferation and survival and is involved in synapse formation, function and plasticity. In cell signal transduction, the $\mathrm{CaN}$ activated by $\mathrm{Ca}^{2+}$ and $\mathrm{CaM}$ was necessary for the dephosphorylation of DAPK1 in neural cell apoptosis (49-51), so the activation of DAPK1 was required the $\mathrm{CaN}$. Fortunately, $\mathrm{CaN}$ is the target protein of the immunosuppressant FK506 (52). In the present study, the authors hypothesized the neuronal cell apoptosis observed following the induction of experimental DAI, and its possible association with the upregulation of DAPK1. FK506 was able to inhibit the activity of DAPK1. Ameliorating the effect of FK506 primarily reduced the amount of neuronal cell death 


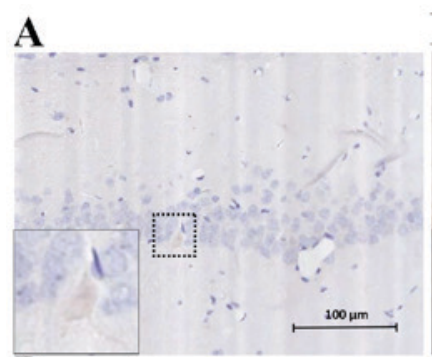

B

C
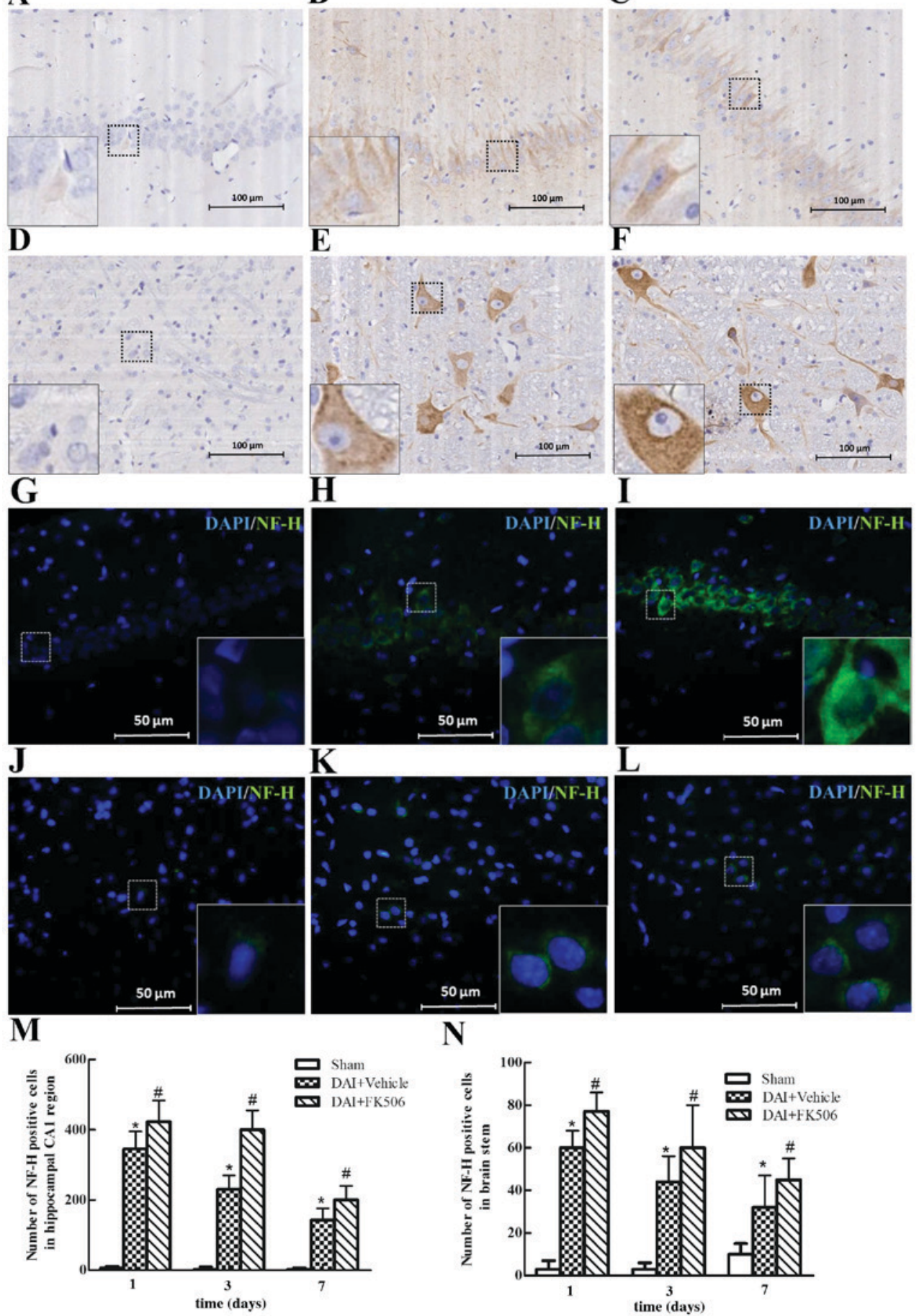

Figure 4. Immunohistochemistry (magnification, x200) and immunofluorescence (magnification, $\mathrm{x} 400$ ) of NF-H in the hippocampal CA1 region and brainstem. Photographs of NF-H immunohistochemistry staining in the hippocampal CA1 region in the (A) Sham (B) DAI+Vehicle and (C) DAI+FK506 groups. Photographs of NF-H immunohistochemistry staining in the brainstem region in the (D) Sham (E) DAI+Vehicle and (F) DAI+FK506 groups. Photographs of NF-H immunofluorescence staining in the hippocampal CA1 region in the (G) Sham (H) DAI+Vehicle and (I) DAI+FK506 groups. Photographs of NF-H immunofluorescence staining in the brainstem region in the (J) Sham (K) DAI+Vehicle and (L) DAI+FK506 groups. Quantitative analysis of the number of NF-H-positive cells $/ \mathrm{mm}^{2}$ in the (M) hippocampal CA1 region and (N) brain stem in Sham DAI+Vehicle and DAI+FK506 groups at 3 days post-injury. ${ }^{*} \mathrm{P}<0.05$ vs. Sham group; ${ }^{\text {}} \mathrm{P}<0.05$ vs. DAI+Vehicle group. NF-H, neurofilament-H; DAI, diffuse axonal injury; DAPK1, death-associated protein kinase 1.

and the expression of DAPK1. Therefore, FK506 was able to inhibit the activation and the expression of DAPK1 and decrease neuronal cell death, which serves a crucial role in neuroprotection following DAI. The process began as early as 1 day post-injury, which indicated that FK506 could inhibit the neuronal apoptosis in the early stage of DAI injury.
NF-H is a structural protein that constitutes a skeletal structure of the nervous cell body and axis, and it may be used as an indicator of the function of the neuron and an indirect indicator of axon regeneration (53). NF-H only exists in the axon under physiological conditions, not in the neuron body. However, the neuron induces a large amount of synthesis of 
A
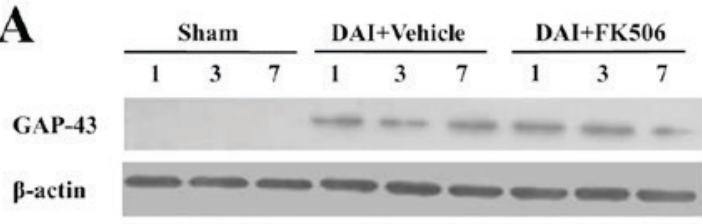

C

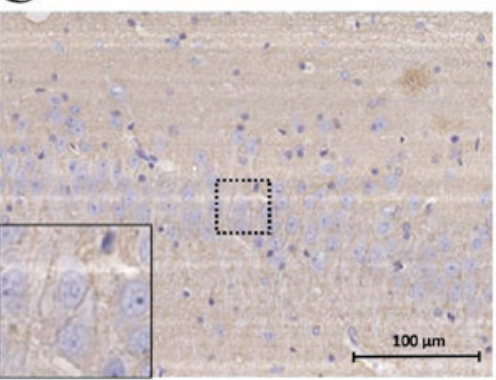

$\mathbf{F}$

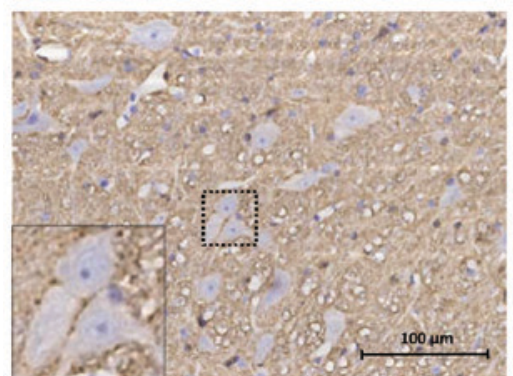

I

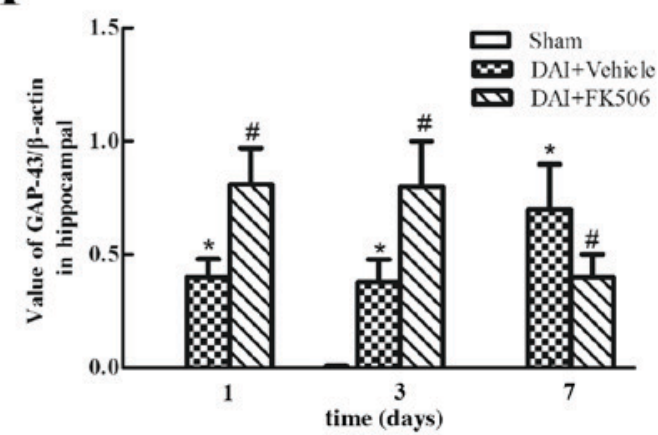

G

\section{B}

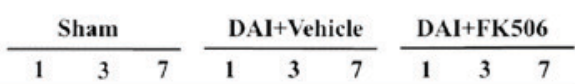

GAP-43

$\beta$-actin

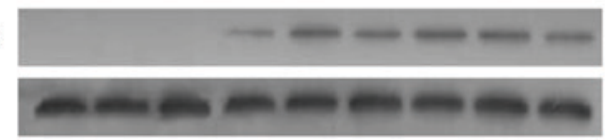

$\mathbf{E}$
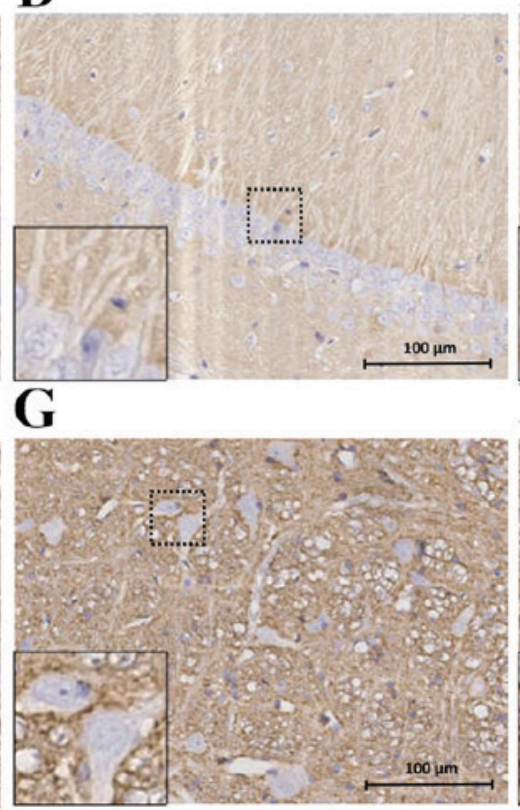

$\mathbf{J}$

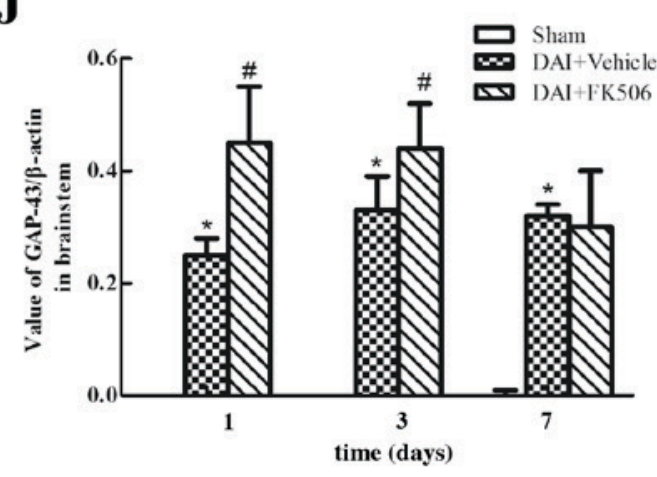

Figure 5. Western blot and immunohistochemistry of GAP-43 in the hippocampal CA1 region and brainstem (magnification, $\mathrm{x} 200$ ). Analysis of GAP-43 in the (A) hippocampal CA1 region and (B) brain stem at each time point following DAI (1, 3 and 7 days). Photographs of GAP-43 staining in the hippocampal CA1 region in (C) Sham, (D) DAI+Vehicle and (E) DAI+FK506 groups. Photographs of GAP-43 immunohistochemistry staining in the brainstem in (F) Sham, (G) DAI+Vehicle and (H) DAI+FK506 groups. Quantitative analysis of the GAP43 in the (I) hippocampal CA1 region and (J) brainstem in Sham, DAI+Vehicle and DAI+FK506 groups at 1,3 and 7 days post-injury. ${ }^{*} \mathrm{P}<0.05$ vs. Sham group; ${ }^{\#} \mathrm{P}<0.05$ vs. DAI+Vehicle group. GAP-43, growth-associated protein-43; DAI, diffuse axonal injury.

NF-H following the TBI, in order to adapt to the demand of nerve regeneration (31). This reflects the neural function and may reflect the condition of axon regeneration. In our study, the expression of NF-H in the FK506-intervention group was significantly increased than that in DAI group, and the peak expression appeared in advance and lasted longer. FK506 may have increased the synthesis of NF-H, accelerated the regeneration of nerve cells, and promoted the repair of nerve tissue following DAI. The mechanism may be associated with the decrease of $\mathrm{T}$ lymphocyte infiltration and destruction, and the inhibition of the apoptosis of neurons. Furthermore, in the present study, immunohistochemistry was carried out on NF-H to identify the pathological alterations to axons following DAI, especially in the early stage of 1 day post-injury. There was a large amount of axonal swelling and axonal retraction ball formation in the damaged regions of the hippocampus and brainstem following DAI. In the FK506-intervention groups, the pathological alterations to axons were markedly improved. Although how the precise mechanism of this axonal protection occurs is not well understood, it has been assumed that FK506 may act by reducing mitochondrial permeability transition, inhibiting CaN activity (22) and increasing the amount of the active form of the GAP-43 in neurons (54).

GAP-43 is a form of nervous tissue-specific protein that is highly expressed in neurons during development and nerve regeneration, which has proven to be implicated in neurite 
outgrowth, long-term potentiation, signal transduction and neurotansmitter release $(28,55)$. In addition, GAP-43 is a rapid transport membrane phosphoric acid protein found in the growth cones of developing and sprouting CNS axons, which is associated with neuronal sprouting, development, differentiation and regeneration $(29,56)$. In the present study, GAP-43 participated in the process of CNS plasticity in response to injury, which is a complex process involving synaptic stability and axonal remodeling, and the level of GAP-43 is additionally predicted to be upregulated in any process that involves axonal membrane remodeling following injury $(29,57,58)$. Therefore, the present study used GAP-43 as a marker of axonal regeneration, and observed that the expression of GAP-43 was remarkably increased at day 1 and gradually increased to a peak at day 3 or 7 , which indicated the altering process of neuronal regeneration following DAI. Hence, GAP-43 may be synthesized in large amounts following DAI, and then shuttled along the axon by fast axonal transport to the site of injury to promote axonal regeneration (56). Although increased expression of GAP-43 has been identified in a DAI rat model in the study, the mechanisms underlying the overexpression of GAP-43 remain unclear. It was observed that in the FK506 intervention group, the expression of GAP-43 was higher than in the DAI group at the same point-in-time, and the peak expression of GAP-43 was observed relatively earlier, at day 1 post-injury. It follows that FK506 promoted a large number of GAP-43 protein synthesis at the injured neurons, and transported from the neuronal bodies to axons, which promoted the regeneration of axons, and accelerate the recovery of nerve function. The upregulation of GAP-43 by FK506 may be associated with the inhibition of $\mathrm{CaN}$ activation and promotion of GAP-43 moving from the binding domain of $\mathrm{CaN}$ to the injured axons $(59,60)$.

In conclusion, the results of the present study indicated that neuronal cell apoptosis and axonal degeneration were observed following experimental DAI. FK506 may serve an important role in anti-apoptosis and attenuating axonal degeneration in experimental DAI by inhibiting the activity of DAPK1. Novel data is provided to suggest that FK506 may promote the axon formation and nerve regeneration after experimental DAI by observing the expressions of GAP-43 and NF-H. Collectively, we summarized that FK506 could reduce the apoptosis of neurons and axon pathological changes following DAI, and relieve the nerve tissue injury. In addition, FK506 may accelerate the regeneration of neurons, and promote the repair of injured brain tissue. However, a study of the precise molecular mechanism of this process remains elusive and further experiments are required to address this aspect.

\section{Acknowledgements}

The present study was supported by the National Natural Science Foundation of China (grant no. 30471774) and the New Century Excellent Talent Support Project of Ministry of Education (grant no. NCET-05-0831).

\section{References}

1. Smith DH, Hicks R and Povlishock JT: Therapy development for diffuse axonal injury. J Neurotrauma 30: 307-323, 2013.
2. Johnson VE, Stewart W and Smith DH: Axonal pathology in traumatic brain injury. Exp Neurol 246: 35-43, 2013.

3. Smith DH, Meaney DF and Shull WH: Diffuse axonal injury in head trauma. J Head Trauma Rehabil 18: 307-316, 2003.

4. Struffert T, Axmann C and Reith W: Craniocerebral trauma. 2: Intra-axial injuries, secondary injuries. Radiologe 43: 1001-1014; quiz 1015-1016, 2003 (In German).

5. Toupalík P,KlírP,Bouska I and ChadováL:Immunohistochemical methods in the differential diagnosis of primary traumatic and subsequent secondary cerebral changes. Soud Lek 45: 18-21, 2000 (In Czech).

6. Tavanti F, Coppola V, Romano A, Beccia M, Giuliani G, Pierallini A and Bozzao A: Diffuse axonal injury with selective involvement of the corticospinal tract. A diffusion tensor imaging case study. Neuroradiol J 27: 397-399, 2014.

7. Jing G, Yao X, Li Y, Xie Y, Li WX, Liu K, Jing Y, Li B, Lv Y and Ma B: Mild hypothermia for treatment of diffuse axonal injury: A quantitative analysis of diffusion tensor imaging. Neural Regen Res 9: 190-197, 2014.

8. Kwon HG and Jang SH: The usefulness of diffusion tensor imaging in detection of diffuse axonal injury in a patient with head trauma. Neural Regen Res 7: 475-478, 2012.

9. Babaee A, Eftekhar-Vaghefi SH, Asadi-Shekaari M Shahrokhi N, Soltani SD, Malekpour-Afshar R and Basiri M: Melatonin treatment reduces astrogliosis and apoptosis in rats with traumatic brain injury. Iran J Basic Med Sci 18: 867-872, 2015.

10. Wang JF, Li Y, Song JN and Pang HG: Role of hydrogen sulfide in secondary neuronal injury. Neurochem Int 64: 37-47, 2014

11. Hilton GD, Stoica BA, Byrnes KR and Faden AI: Roscovitine reduces neuronal loss, glial activation, and neurologic deficits after brain trauma. J Cereb Blood Flow Metab 28: 1845-1859, 2008.

12. Ma Y, Liu W, Wang Y, Chao X, Qu Y, Wang K and Fei Z: VEGF protects rat cortical neurons from mechanical trauma injury induced apoptosis via the MEK/ERK pathway. Brain Res Bull 86: 441-446, 2011.

13. Hardingham GE and Bading H: Synaptic versus extrasynaptic NMDA receptor signalling: Implications for neurodegenerative disorders. Nat Rev Neurosci 11: 682-696, 2010.

14. Karklin Fontana AC, Fox DP,Zoubroulis A, Valente Mortensen O and Raghupathi R: Neuroprotective effects of the glutamate transporter activator (R)-(-)-5-methyl-1-nicotinoyl-2-pyrazoline (MS-153) following traumatic brain injury in the adult rat. J Neurotrauma 33: 1073-1083, 2016.

15. Armstrong RC, Mierzwa AJ, Marion CM and Sullivan GM: White matter involvement after TBI: Clues to axon and myelin repair capacity. Exp Neurol 275: 328-333, 2016.

16. Tang-Schomer MD, Patel AR, Baas PW and Smith DH: Mechanical breaking of microtubules in axons during dynamic stretch injury underlies delayed elasticity, microtubule disassembly and axon degeneration. FASEB J 24: 1401-1410, 2010.

17. Brizuela M, Blizzard CA, Chuckowree JA, Dawkins E, Gasperini RJ, Young KM and Dickson TC: The microtubule-stabilizing drug Epothilone D increases axonal sprouting following transection injury in vitro. Mol Cell Neurosci 66: 129-140, 2015.

18. Zawadzka $M$ and Kaminska B: A novel mechanism of FK506-mediated neuroprotection: Downregulation of cytokine expression in glial cells. Glia 49: 36-51, 2005.

19. Shichinohe H, Kuroda S, Abumiya T, Ikeda J, Kobayashi T, Yoshimoto $\mathrm{T}$ and Iwasaki Y: FK506 reduces infarct volume due to permanent focal cerebral ischemia by maintaining BAD turnover and inhibiting cytochrome c release. Brain Res 1001: 51-59, 2004.

20. Gabryel B, Bielecka A, Stolecka A, Bernacki J and Langfort J: Cytosolic phospholipase $\mathrm{A}_{2}$ inhibition is involved in the protective effect of nortriptyline in primary astrocyte cultures exposed to combined oxygen-glucose deprivation. Pharmacol Rep 62: 814-826, 2010.

21. Singleton RH, Stone JR, Okonkwo DO, Pellicane AJ and Povlishock JT: The immunophilin ligand FK506 attenuates axonal injury in an impact-acceleration model of traumatic brain injury. J Neurotrauma 18: 607-614, 2001.

22. Marmarou CR and Povlishock JT: Administration of the immunophilin ligand FK506 differentially attenuates neurofilament compaction and impaired axonal transport in injured axons following diffuse traumatic brain injury. Exp Neurol 197: 353-362, 2006. 
23. Reeves TM, Phillips LL, Lee NN and Povlishock JT: Preferential neuroprotective effect of tacrolimus (FK506) on unmyelinated axons following traumatic brain injury. Brain Res 1154: 225-236, 2007.

24. Tu W, Xu X, Peng L, Zhong X, Zhang W, Soundarapandian MM, Balel C, Wang M, Jia N, Zhang W, et al: DAPK1 interaction with NMDA receptor NR2B subunits mediates brain damage in stroke. Cell 140: 222-234, 2010.

25. Bialik S and Kimchi A: The death-associated protein kinases: Structure, function, and beyond. Annu Rev Biochem 75: 189-210, 2006.

26. Pelled D, Raveh T, Riebeling C, Fridkin M, Berissi H, Futerman AH and Kimchi A: Death-associated protein (DAP) kinase plays a central role in ceramide-induced apoptosis in cultured hippocampal neurons. J Biol Chem 277: 1957-1961, 2002.

27. Kato S, Matsukawa T, Koriyama Y, Sugitani K and Ogai K: A molecular mechanism of optic nerve regeneration in fish: The retinoid signaling pathway. Prog Retin Eye Res 37: 13-30, 2013.

28. Liu F, Liao F, Li W, Han Y and Liao D: Progesterone alters Nogo-A, GFAP and GAP-43 expression in a rat model of traumatic brain injury. Mol Med Rep 9: 1225-1231, 2014.

29. Benowitz LI and Routtenberg A: GAP-43: An intrinsic determinant of neuronal development and plasticity. Trends Neurosci 20 : 84-91, 1997.

30. Zhang YM, Zhang YQ, Cheng SB, Chen SX, Chen AL and Tang CZ: Effect of acupuncture on proliferation and differentiation of neural stem cells in brain tissues of rats with traumatic brain injury. Chin J Integr Med 19: 132-136, 2013.

31. Zurek J and Fedora M: The usefulness of S100B, NSE, GFAP, $\mathrm{NF}-\mathrm{H}$, secretagogin and Hsp70 as a predictive biomarker of outcome in children with traumatic brain injury. Acta Neurochir (Wien) 154: 93-103, 2012.

32. Li Y, Song J, Liu X, Zhang M, An J, Sun P, Li D, Jin T and Wang J: High expression of STIM1 in the early stages of diffuse axonal injury. Brain Res 1495: 95-102, 2013.

33. Xiao-Sheng H, Sheng-Yu Y, Xiang Z, Zhou F and Jian-ning Z: Diffuse axonal injury due to lateral head rotation in a rat model. J Neurosurg 93: 626-633, 2000.

34. Xiao-Sheng H, Gui-Tao Y, Xiang Z and Zhou F: A morphological study of diffuse axonal injury in a rat model by lateral head rotation trauma. Acta Neurol Belg 110: 49-56, 2010.

35. Marsland TA, Glees P and Erikson LB: Modification of the Glees silver impregnation for paraffin sections. J Neuropathol Exp Neurol 13: 587-591, 1954.

36. Ng HK, Mahaliyana RD and Poon WS: The pathological spectrum of diffuse axonal injury in blunt head trauma: Assessment with axon and myelin strains. Clin Neurol Neurosurg 96: 24-31, 1994.

37. Maroon H, Walshe J, Mahmood R, Kiefer P, Dickson C and Mason I: Fgf3 and Fgf8 are required together for formation of the otic placode and vesicle. Development 129: 2099-2108, 2002

38. Jia X, Cong B, Wang S, Dong L, Ma C and Li Y: Secondary damage caused by $\mathrm{CD} 11 \mathrm{~b}+$ microglia following diffuse axonal injury in rats. J Trauma Acute Care Surg 73: 1168-1174, 2012.

39. Logsdon AF, Lucke-Wold BP, Turner RC, Huber JD, Rosen CL and Simpkins JW: Role of microvascular disruption in brain damage from traumatic brain injury. Compr Physiol 5: 1147-1160, 2015.

40. Dressler J and Vemuganti R: Apoptosis and gene expression after TBI. Leg Med (Tokyo) 11 (Suppl 1): S54-S55, 2009.

41. Mao Z, Song Z, Li G, Lv W, Zhao X, Li B, Feng X and Chen Y: 8-hydroxy-2-(di-n-propylamino)tetralin intervenes with neural cell apoptosis following diffuse axonal injury. Neural Regen Res 8: 133-142, 2013.

42. Aberg M, Johnell M, Wickstrom M and Siegbahn A: Tissue Factor/FVIIa prevents the extrinsic pathway of apoptosis by regulation of the tumor suppressor Death-Associated Protein Kinase 1 (DAPK1). Thromb Res 127: 141-148, 2011.
43. Yoo HJ, Byun HJ, Kim BR, Lee KH, Park SY and Rho SB: DAPk1 inhibits NF- $\mathrm{BB}$ activation through TNF- $\alpha$ and INF- $\gamma$-induced apoptosis. Cell Signal 24: 1471-1477, 2012.

44. Martoriati A, Doumont G, Alcalay M, Bellefroid E, Pelicci PG and Marine JC: dapk1, encoding an activator of a p19ARF-p53-mediated apoptotic checkpoint, is a transcription target of p53. Oncogene 24: 1461-1466, 2005.

45. Pei L, Shang Y, Jin H, Wang S, Wei N, Yan H, Wu Y, Yao C, Wang X, Zhu LQ and Lu Y: DAPK1-p53 interaction converges necrotic and apoptotic pathways of ischemic neuronal death. J Neurosci 34: 6546-6556, 2014

46. Tian J, Cheng J, Zhang J, Ye L, Zhang F, Dong Q, Wang H and $\mathrm{Fu} F$ : Protection of pyruvate against glutamate excitotoxicity is mediated by regulating DAPK1 protein complex. PLoS One 9: e95777, 2014.

47. Lai TW, Zhang S and Wang YT: Excitotoxicity and stroke: Identifying novel targets for neuroprotection. Prog Neurobiol 115: 157-188, 2014.

48. Shamloo M, Soriano L, Wieloch T, Nikolich K, Urfer R and Oksenberg D: Death-associated protein kinase is activated by dephosphorylation in response to cerebral ischemia. J Biol Chem 280: 42290-42299, 2005.

49. Fujita Y and Yamashita T: Role of DAPK in neuronal cell death Apoptosis 19: 339-345, 2014.

50. Nair S, Hagberg H, Krishnamurthy R, Thornton C and Mallard C: Death associated protein kinases: Molecular structure and brain injury. Int J Mol Sci 14: 13858-13872, 2013.

51. Schumacher AM, Velentza AV and Watterson DM Death-associated protein kinase as a potential therapeutic target. Expert Opin Ther Targets 6: 497-506, 2002.

52. Wang HL, Du YW, Xiang BQ, Lin WL and Wei Q: The regulatory domains of CNA have different effects on the inhibition of CN activity by FK506 and CsA. IUBMB Life 59: 388-393, 2007.

53. Shaw G, Yang C, Ellis R, Anderson K, Parker Mickle J, Scheff S, Pike B, Anderson DK and Howland DR: Hyperphosphorylated neurofilament NF-H is a serum biomarker of axonal injury. Biochem Biophys Res Commun 336: 1268-1277, 2005.

54. Bavetta S, Hamlyn PJ, Burnstock G, Lieberman AR and Anderson PN: The effects of FK506 on dorsal column axons following spinal cord injury in adult rats: Neuroprotection and local regeneration. Exp Neurol 158: 382-393, 1999.

55. Huang HL, Li JM and Zhao YN: Effect of shenxiong huayu capsule on cerebral ischemia/reperfusion injury and the expression of GAP43 in hippocampal CA1 of rats. Zhongguo Zhong Xi Yi Jie He Za Zhi 34: 185-190, 2014 (In Chinese).

56. Williams RR, Venkatesh I, Pearse DD, Udvadia AJ and Bunge MB: MASH1/Asclla leads to GAP43 expression and axon regeneration in the adult CNS. PLoS One 10: e0118918, 2015.

57. Hulsebosch CE, DeWitt DS, Jenkins LW and Prough DS Traumatic brain injury in rats results in increased expression of Gap-43 that correlates with behavioral recovery. Neurosci Lett 255: 83-86, 1998.

58. Li H, Dokas LA, Godfrey DA and Rubin AM: Remodeling of synaptic connections in the deafferented vestibular nuclear complex. J Vestib Res 12: 167-183, 2002.

59. Baumgärtel K and Mansuy IM: Neural functions of calcineurin in synaptic plasticity and memory. Learn Mem 19: 375-384, 2012.

60. Boczek T, Ferenc B, Lisek M and Zylinska L: Regulation of GAP43/calmodulin complex formation via calcineurin-dependent mechanism in differentiated PC12 cells with altered PMCA isoforms composition. Mol Cell Biochem 407: 251-262, 2015. 